\title{
Improved inclination correction method applied to the guidance system of agricultural vehicles
}

\author{
Ricardo Ospina, Noboru Noguchi* \\ (Research Faculty of Agriculture, Hokkaido University, Sapporo 060-8589 Hokkaido, Japan)
}

\begin{abstract}
This research introduces a new inclination correction method with increased accuracy applied to the guidance system of an agricultural vehicle. The method considers the geometry of a robot tractor and uses an Inertial Measurement Unit (IMU) to correct the lateral error of the RTK-GPS antenna measurements raised by the tractor's inclinations. A parameters optimization experiment and an automatic guidance experiment under real working conditions were used to compare the accuracy of a traditional correction method with the new correction method, by calculating the RMSE of the lateral error and the error reduction percentage. An additional tuned correction method was found by using a simple analytical method to find the optimal variables that reduced the lateral error to a minimum. The results indicate that the new correction method and the tuned correction method display a significant error reduction percentage compared to the traditional correction method. The methods could correct the GPS lateral error caused by the roll inclinations in real-time. The resulting lateral deviation caused by the tractor's inclinations could be reduced up to $23 \%$ for typical travelling speeds.
\end{abstract}

Keywords: inclination correction method, guidance system, agricultural vehicles, robot tractor, IMU, RTK-GPS, precision farming

DOI: $10.25165 /$ j.ijabe.20201306.6012

Citation: Ospina R, Noguchi N. Improved inclination correction method applied to the guidance system of agricultural vehicles. Int J Agric \& Biol Eng, 2020; 13(6): 183-194.

\section{Introduction}

Aging and population decline have been progressing in Japanese society ${ }^{[1]}$, and this tendency is more significantly seen in rural areas than in urban areas. To protect food production in Japan, the development of technologies such as autonomous guidance systems for agricultural vehicles is an effective strategy to deal with the gradually decreasing farming labor force, in addition, to increase production efficiency and operation safety ${ }^{[2]}$. As a result, position and information technologies are changing the farmer's relationship with their land.

Researchers and manufacturers are emphasizing machine control systems to minimize the time required for operation and ease the hard-monotonous routine work associated with field operations. The autonomous guidance systems for agricultural vehicles, such as tractors, are designed to provide drivability over fields and off-road surfaces. Therefore, the accuracy and precision of these type of systems is a critical factor ${ }^{[3]}$. This kind of system usually includes a navigation controller, actuators for driving control, positioning of the vehicle by GPS, an attitude measurement by $\mathrm{IMU}^{[4]}$. Since the positioning of agricultural vehicles depends mainly on GPS based technologies, measurement of the GPS data requires to know where the antenna is situated in the vehicle, and typically this is in the center of the cab roof ${ }^{[5]}$, over $2 \mathrm{~m}$ height from the ground. This installation setup causes a position error resulting from the vehicle's inclinations in the roll

Received date: 2020-07-09 Accepted date: 2020-10-18

Biographies: Ricardo Ospina, JSAM Member, PhD, Postdoctoral Fellow, research interest: agricultural robots, vehicle robotics, Email: rospina@, bpe.agr.hokudai.ac.jp.

*Corresponding author: Noboru Noguchi, JSAM Member, PhD, Professor, research interest: agricultural robots, vehicle robotics. Research Faculty of Agriculture, Hokkaido University, Kita-9, Nishi-9, Kita-Ku, Sapporo, Hokkaido, 060-8589, Japan. Tel: +81-11-7063847, Email: noguchi@bpe.agr.hokudai.ac.jp. and pitch directions. Therefore, a correction method of the position error caused by the vehicle's inclinations must be considered in the GPS antenna measurements.

For ground vehicles, the traditional correction method consists of using Eulerian angles and the distance from the GPS antenna to the vehicle's center of gravity to estimate the rotation in the 3 spatial dimensions, assuming that the vehicle body rotates around the center of gravity as a fixed point ${ }^{[6]}$. This approach has acceptable accuracy for on-road vehicles ${ }^{[7]}$, particularly to estimate the inclination caused by the road grade. However, for an agricultural vehicle such as a tractor, this approach poses 2 problems.

The first and more common problem is the estimation of the tractor's center of gravity. The experimental setup for measuring the center of gravity of a tractor is quite complex and requires specialized equipment ${ }^{[8]}$. For agricultural vehicles, since the estimation of a vehicle's center of gravity poses more problems than it solves, an approximation using the weight of the front and rear axles ${ }^{[9]}$ is a simple yet acceptable approach if demanding dynamics such as high speed, mass changes or sudden steering maneuvers are not a requirement ${ }^{[10]}$. In the case of tractors, a weighting $\operatorname{method}^{[11]}$ or an alternative method $^{[12]}$ using trigonometric functions can give an approximation of the tractor's center of gravity but such approaches have their limitations, like requiring specialized equipment or software and using procedures that are complicated and time-consuming.

The second problem is that for a four-wheeled tractor the rotation in the roll and pitch directions does not occur exactly around the tractor's center of gravity. Such consideration is correct for an aerial vehicle ${ }^{[13]}$ and it is a good approximation for a four-wheeled on-road vehicle ${ }^{[14]}$, but it is necessary to consider that the height of the rooftop and the suspension of an on-road vehicle are different compared to the average tractor. It is important to note that the common tractors usually have a suspension system 
that is too rigid ${ }^{[15]}$ compared to an on-road vehicle. In some cases, the tractors do not have a tire suspension system at all ${ }^{[16]}$, the suspension is installed in the driver's seat or the cabin. Therefore, the support provided by the tractor's four wheels is rigid and the rotation around the roll direction does not occur around the tractor's center of gravity, it occurs around the tires acting as a pivot point. As a result, the center of gravity does not behave as a fixed point, it moves in the rotation direction. This situation is described in detail in Section 2.2. This situation combined with the tractor's rooftop larger height causes an amount of error when using the traditional correction method around the center of gravity. For real agricultural work, this amount of error is always present, and it also depends on the slope and roughness of the terrain.

Several researchers have worked on RTK-GPS-based guidance systems that offer an acceptable level of accuracy for autonomous steering during agricultural operations by using an inclination correction method. For example, an implemented algorithm to compensate for GPS position errors that occur when a vehicle travels on inclined planes ${ }^{[17]}$. In principle, similar to the method used in commercial GPS products with terrain compensation, such as Trimble T3 (Sunnyvale, CA, USA) and John Deere iTC (Moline, IL, USA), the GPS position errors are corrected by performing Euler rotations based on real-time measurement of vehicle orientation (roll, pitch, and heading angles) obtained with an inertial measurement unit (IMU) sensor mounted on the tractor. Other examples of the use of the traditional inclination correction $\operatorname{method}^{[18]}$ can give as a result a lateral error of less than about $0.2 \mathrm{~m}$. However, results indicate that the error is large at begin and end of the inclination. As a solution to this problem, estimation of the position in real-time by using a Kalman filter is proposed. Other researchers ${ }^{[19]}$ have taken advantage of this traditional inclination correction in combination with sensor fusion techniques to improve the position estimated over the accuracy of a DGPS; thus, enabling to guide an agricultural vehicle autonomously along the target line in the field. However, the use of the traditional inclination correction method applied to the automatic guidance system of an agricultural vehicle still poses the two problems described above. It is still necessary to improve the performance of the previously developed inclination correction methods.

Therefore, the purpose of this research was to develop an improved inclination correction method that considers the rotation around the tires acting as a pivot point in order to improve the accuracy of the automatic guidance system of a robot tractor. This factor constitutes the originality of this research.

\section{Materials and methods}

\subsection{Experimental platform}

The experimental vehicle is a robot tractor (EG-83, Yanmar Co., Japan) equipped with an on-board computer that logs the data from the sensors. The robot tractor supports the control of the hitch function, the power-take-off, the engine speed, the transmission, as well as the steering angle during autonomous operation. This control is based on postural information from the RTK-GPS and IMU in reference to a navigation map. All stages of fieldwork including tillage, seeding, spraying and harvesting, can be automated within an error of $5 \mathrm{~cm}$ when the robot is equipped with a map of its travel path ${ }^{[20]}$. Figure 1 shows the experimental vehicle and the sensors equipped for the experiment.

The RTK-GPS (SPS855, Trimble, US) provides the position, direction of travel and speed of the vehicle. The low latency configuration (update rate: $10 \mathrm{~Hz}$, latency: $0.02 \mathrm{~s}$ ) was chosen for the RTK mode. This configuration provides a horizontal position accuracy of $2 \mathrm{~cm}+2 \mathrm{~mm} / \mathrm{km}$, vertical position accuracy of $3 \mathrm{~cm}+$ $2 \mathrm{~mm} / \mathrm{km}$ and speed accuracy of $0.16 \mathrm{~km} / \mathrm{h}$. The RTK correction signal was obtained using a Virtual Reference System via an Internet Service Provider connected to the on-board computer that logs the data from the GPS receiver. The inertial measurement unit (IMU) (VN100, VectorNav, US) provides the vehicle's attitude angles (dynamic accuracy: 2.0 ${ }^{\circ}$. RMS). Both the GPS and the IMU have a direct serial port connection to the on-board computer. The speed of all serial connections was $115200 \mathrm{bit} / \mathrm{s}$. However, since the GPS NMEA data messages and the IMU data messages have different lengths, all the data was synchronized using the computer's timestamp. The result was a measurement update rate of $10 \mathrm{~Hz}$ for all the sensors. The experiments were conducted at the experimental farm of Hokkaido University, Hokkaido, Japan, between April 2020 and June 2020. The experimental location was an open sky environment, meaning there were no sight-blocking buildings or trees.

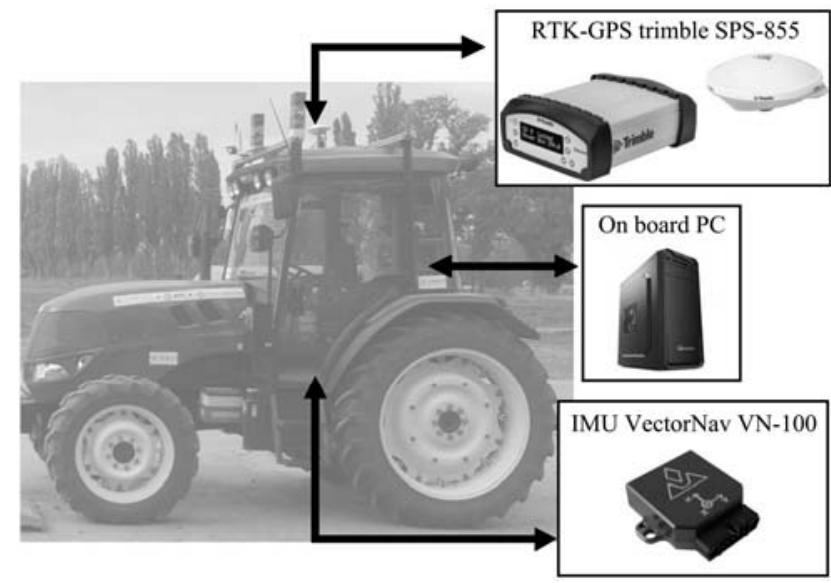

Figure 1 Experimental vehicle and equipped sensors

\subsection{Inclination correction of the GPS position}

For robot tractor applications, it is necessary to get an accurate vehicle position for the automatic guidance system. Typically, the GPS antenna is installed at the rooftop of the robot tractor in order to receive enough satellite signals ${ }^{[21]}$. However, the GPS antenna's height respect to the ground causes a position error raised from the tractor's inclinations in the roll and pitch directions.

As expected, this positioning error increases if the GPS antenna's height and the tractor's roll angle increase. The error caused by the tractor's inclination can be corrected using Eulerian angles ${ }^{[22]}$; the corrected position of the GPS antenna under the $\mathrm{X}-Y$-coordinate system is described by Equation (1).

$$
\left(\begin{array}{c}
X_{a} \\
Y_{a} \\
Z_{a}
\end{array}\right)=\left(\begin{array}{c}
X \\
Y \\
Z
\end{array}\right)-E^{-1}\left(\varphi, \theta_{p}, \theta_{r}\right) \cdot\left(\begin{array}{l}
a \\
b \\
h
\end{array}\right)
$$

where, $X_{a}, Y_{a}, Z_{a}$ represent corrected position from the GPS antenna, $\mathrm{m} ; X, Y, Z$ represent antenna position acquired by the GPS, $\mathrm{m} ; a, b$, $h$ represent distances from the center of gravity to the GPS antenna, $\mathrm{m} ; \varphi$ is the yaw angle measured by the IMU, $\left(^{\circ}\right) ; \theta_{p}$ is the pitch angle measured by the IMU, $\left({ }^{\circ}\right) ; \theta_{r}$ is the roll angle measured by the IMU, $\left({ }^{\circ}\right)$.

The matrix $E$ from Equation (1) is the transformation matrix of the tractor's coordinate system to the $X-Y$ coordinate system. It is described in detail by Equation (2) as: 


$$
E^{-1}\left(\varphi, \theta_{p}, \theta_{r}\right)=E\left(-\varphi,-\theta_{p},-\theta_{r}\right)=\left(\begin{array}{ccc}
\cos \theta_{r} \cos \varphi+\sin \theta_{r} \sin \theta_{p} \sin \varphi & \cos \theta_{p} \sin \varphi & -\cos \theta_{r} \sin \theta_{p} \sin \varphi+\sin \theta_{r} \cos \varphi \\
-\cos \theta_{r} \sin \varphi+\sin \theta_{r} \sin \theta_{p} \cos \varphi & \cos \theta_{p} \cos \varphi & -\cos \theta_{r} \sin \theta_{p} \cos \varphi-\sin \theta_{r} \sin \varphi \\
-\sin \theta_{r} \cos \theta_{p} & \sin \theta_{p} & \cos \theta_{r} \cos \theta_{p}
\end{array}\right)
$$

Consider in Equation (2) the situation when both the yaw angle $\varphi$ and the roll angle $\theta_{r}$ are zero. In this situation, the positioning error raised by the pitch angle $\theta_{p}$ causes a longitudinal deviation along the tractor's direction of travel. This means that the position measured by the GPS antenna is ahead of the real position along the direction of travel if the pitch $\theta_{p}$ is positive or behind the real position if the pitch $\theta_{p}$ is negative. Please note that the positioning error caused by the pitch angle $\theta_{p}$ will be parallel to the direction of travel. For a real agriculture task, most of the crop rows are parallel because of the disposition of the agricultural implements. Therefore, the inclination correction of the GPS position error caused by the pitch $\theta_{p}$ is not critical for the performance of a tractor's automated guidance system.

Now consider in Equation (2) the situation when both the yaw angle $\varphi$ and the pitch angle $\theta_{p}$ are zero. In this situation, the positioning error caused by the roll angle $\theta_{r}$ causes a lateral deviation, perpendicular to the tractor's direction of travel. The correction of the positioning error caused by the roll angle $\theta_{r}$ is critical because it causes a lateral deviation that affects directly the performance of the robot tractor when it travels in the field among the crop rows. For example, if the GPS antenna is installed on the top of the robot tractor $2 \mathrm{~m}$ above the ground and the roll is around $3^{\circ}$, the positioning error between the top of the GPS antenna and the bottom of the antenna is about $0.10 \mathrm{~m}^{[22]}$. This can cause the robot tractor to step in the crop rows and thus spoiling the plants.

Note that the correction in the roll direction depends mainly on the value of the height $h$ from the center of gravity to the GPS antenna. Figure $2^{[23]}$ indicates the roll angle $\theta_{r}$ effect for this inclination interaction; the tractor in the figure is shown from behind. Figure 2a shows the theoretical rotation around the center of gravity; the top figure indicates the fixed center of gravity of a tractor that tilts from its initial horizontal position to a final inclined position while the bottom figure indicates only the final inclined position for clarification. To perform the correction of the GPS antenna by using Equation (1) and Equation (2), it is necessary to measure the distances $a, b$, and $h$ from the tractor's center of gravity to the GPS antenna. This poses an additional problem besides the estimation of the tractor's center of gravity not being a simple process ${ }^{[12]}$ because the tractor does not rotate around its center of gravity as a fixed point aligned with the vertical reference line as displayed in Figure 2a. A typical on-road vehicle has a lower center of gravity, a shorter rooftop height, a wider tire track width and a softer tire suspension compared to a typical tractor; which has a higher center of gravity, and a narrower track width relative to a larger rooftop height. The typical tractor suspension is too stiff or inexistent ${ }^{[15]}$. If the tractor were to rotate around its center of gravity as a fixed point, the tire would sink or pierce into the solid ground. Of course, such a situation is unreal.

Figure $2 \mathrm{~b}$ shows a situation closer to reality; the real rotation around the tire acting as a pivot point. The top figure indicates the shifting center of gravity of a tractor that tilts from its initial horizontal position to a final inclined position, while the bottom figure indicates only the final inclined position for clarification. In this case, the tractor's center of gravity shifts to the right side and upwards. Note that the tractor's right tire stays aligned in the same position, indicated by the dotted line. Therefore, the fixed rotation point is not the tractor's center of gravity, it is the tire's contact point with the ground. That is the reason why the tire acts as a pivot point. Even though, the initial notion from Figure $2 b$ is that performing the inclination correction of the GPS position assuming rotation around the center of gravity as a fixed point will have just a small amount of lateral error because the center of gravity does not shift away from the vertical reference line a significant distance; furthermore this misleads to assume that such error will be close to zero if the tractor's center of gravity is closer to the ground. However, it is necessary to keep in mind that the purpose of the correction is to reduce the lateral error to keep the tractor's tires as parallel as possible to the crop rows ${ }^{[24]}$ to keep the tires away from damaging the plants. Therefore, a better notion would be to perform the inclination correction of the GPS position with respect to the middle point of the tractor's track width.

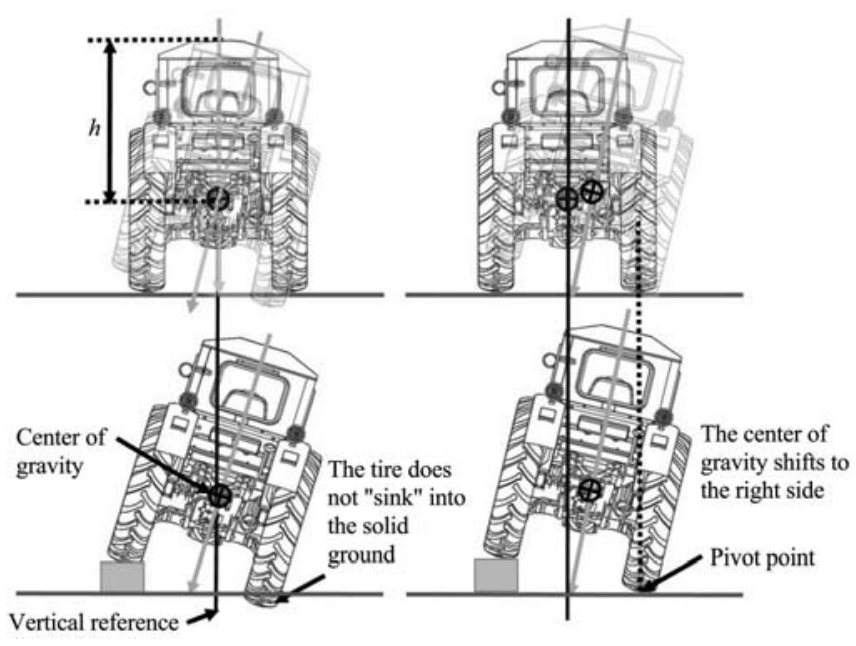

a. Theoretical rotation around the center of gravity

b. Real rotation around the tire

Figure 2 The effect of the roll angle $\theta_{r}$

Figure 3a explains better the traditional method for this inclination correction considerations; the tractor in the figure is shown from behind. Some of the parameters required to perform the inclination correction of the GPS position using Equation (1) and Equation (2) are shown in Figure 3a for a better explanation. Typically, the GPS antenna is installed on the tractor's rooftop ${ }^{[21]}$ in the middle of the track width. The GPS antenna is represented in Figure 3a by the hexagon on the tractor's rooftop. If the tractor is in a horizontal position, the position acquired by the GPS antenna $X$ will coincide with the vertical reference line and the middle of the tractor's track width. If the tractor tilts, the position acquired by the GPS antenna $X$ will shift away from the vertical reference line in the inclination direction, causing the measured position error. By performing the inclination correction of the GPS position using Equation (1) and Equation (2); including the roll angle $\theta_{r}$ measured by the IMU and the height $h$ from the center of gravity $(C O G)$ to the GPS antenna, it is possible to transform $X$ into the corrected position $X_{a}$.

From Figure 3a, it becomes obvious that the corrected position $X_{a}$ depends directly on the value of $h$. If the $C O G$ is low, it is closer to the ground, $h$ is large along the tractor's central reference line, then $X_{a}$ is closer to the middle of the tractor's track width. If the $C O G$ is high it is closer to the rooftop, $h$ is short and then $X_{a}$ is closer to $X$, making the correction meaningless. Therefore, for 
this type of inclination correction of the GPS position, the correct determination of the tractor's $C O G$ is a necessary but not sufficient factor. Even if the tractor's $C O G$ is estimated with high accuracy, this does not guarantee that it will be located closer to the ground, far away from the rooftop along the tractor's central reference line ${ }^{[25]}$ to obtain an inclination correction with a minimum error. Of course, Figure 3a shows a hypothetical situation for a rather large roll angle $\theta_{r}$, but it is necessary to consider that even for a small roll angle $\theta_{r}$ the corrected position $X_{a}$ still depends directly on the value of $h$ and the height of the $C O G$ respect to the ground. For medium-sized tractors the average height is $2.8 \mathrm{~m}^{[26]}$; the taller the tractor, the higher the COG. This means that if the value of $h$ is estimated with high accuracy and happens to be closer to the rooftop than to the tire's baseline in the ground, then $X_{a}$ will always have an amount of error.

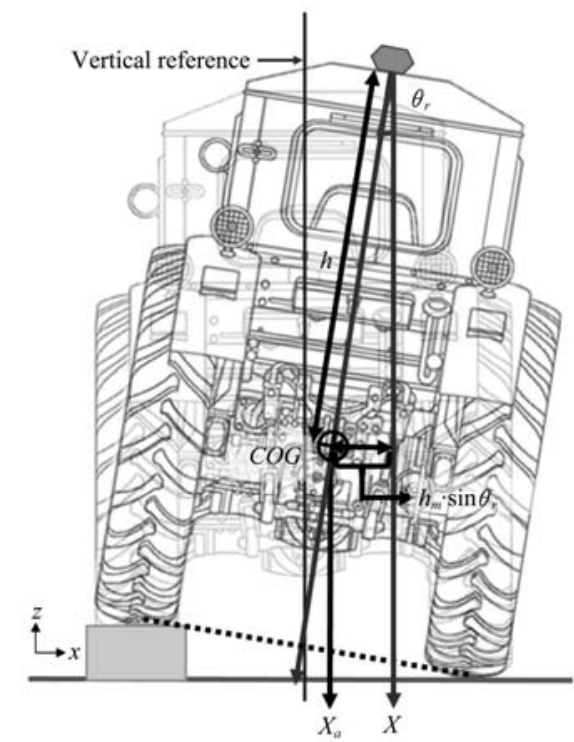

a. Traditional method

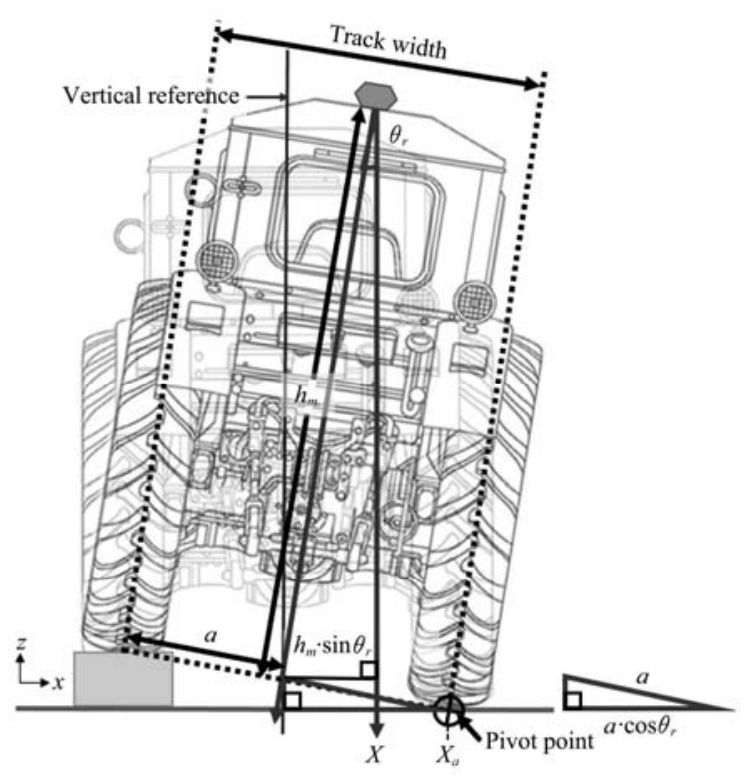

b. New method

Figure 3 Inclination correction considerations

Therefore, to avoid the complexity of the determination of a $C O G$ that is going to introduce an amount of error anyway, this research proposes a simpler and yet more accurate approach. Figure $3 b$ shows the new method's inclination correction considerations. Figure 3b shows the rooftop measured height $h_{m}$. Again, the GPS antenna is represented by the hexagon on the tractor's rooftop and it is placed in the middle of the tractor's track width. The fixed rotation point is the tire's contact point with the ground, indicated as the pivot point. By this simple redefinition of the rotation point, it is possible to use Equation (1) and Equation (2) by also redefining the corrected position $X_{a}$ as the projection of the pivot point onto the horizontal axis.

Please note that in Equation (1) and Figure $3 \mathrm{~b}$ that if $\varphi=\theta_{p}=0$ then $X_{a}=X+a \cdot \cos \theta_{r}-h_{m} \cdot \sin \theta_{r}$; where $a$ is redefined as the lateral distance from the rotation point in the tire's contact point to the GPS antenna and corresponds to half the tractor's track width. Please also note that for the triangles in Figure 3b, the distance $a$ is constant whereas the distances $h_{m} \cdot \sin \theta_{r}$ and $a \cdot \cos \theta_{r}$ of course increase or decrease according to the value of the roll angle $\theta_{r}$. Please remember that $X_{a}$ in both Figure $3 \mathrm{a}$ and Figure $3 \mathrm{~b}$ is the projection over the horizontal axis of the rotation point. For the traditional correction method, shown in Figure $3 \mathrm{a}$, the rotation point is the tractor's center of gravity. For the new correction method, shown in Figure 3b, the rotation point is the tractor's tire acting as a pivot point. By looking at Figure $3 \mathrm{~b}$, it is obvious that $X_{a}$ is the position of the tractor's right tire with respect to the GPS antenna. However, it is more convenient to use the center point of the tractor's track width for the automatic guidance system, because this makes the control and positioning of the machine simpler. Therefore, to perform the inclination correction of the GPS position with respect to the middle point of the tractor's track width, it is possible to use the simplification $X_{a c}=X-h_{m} \cdot \sin \theta_{r}$, which is also indicated in Figure $3 b$. Please remember that the inclination correction of the GPS position error caused by the pitch $\theta_{p}$ is not critical for the performance of a tractor's automated guidance system because the pitch angle $\theta_{p}$ is parallel to the direction of travel.

Measuring the rooftop height $h_{m}$ and the tractor's track width is a simpler and more straightforward process compared to the determination of the tractor's COG; and it does not require complex or specialized tools. This research took advantage of this simplification and compared the experimental results of the traditional correction method with the new correction method. In addition, a simple analytical method was used in a simulation to find the optimal value of $h_{m}$ that produced the smallest amount of error, giving, as a result, a tuned correction method. The next section gives an explanation of the experimental method used in this research.

\subsection{Experimental method}

The experiments were conducted at the experimental farm of Hokkaido University, Hokkaido, Japan, between April 2020 and June 2020. The experimental location was an open sky environment, meaning there were no sight-blocking buildings or trees. Two main experiments were performed in this research.

\subsubsection{Parameters optimization}

To perform the parameters optimization an experiment was carried out on a flat, horizontal surface made of cast concrete. The purpose of the experiment was to verify the effect of the roll angle in the position error when the tractor was traveling at an approximately constant speed. Two speeds were tested in this experiment, $0.6 \mathrm{~m} / \mathrm{s}$ and $3.0 \mathrm{~m} / \mathrm{s}$. These two values were selected because they were close to the typical minimum and maximum speed values of the tractor in working conditions in the field and traveling conditions on the road ${ }^{[16]}$. This test allowed to compare the accuracy of the traditional correction method to the accuracy of the new correction method. More important, it also allowed the selection and optimization of the parameters in Equation (1) in 
order to minimize the lateral error and produce a tuned correction method. The first part of the experiment consisted of driving the tractor manually on the flat surface without providing any inclinations; i.e. in horizontal condition. The tractor was driven following a straight path of $7 \mathrm{~m}$ length while logging both GPS and IMU data. The experimental straight path was measured by using reference lines drawn in the horizontal concrete surface. This allowed driving the tractor along the same longitudinal path for both the horizontal condition and the inclined condition. Therefore, the straight path is used as the reference path; the GPS and IMU data from the manual drive in horizontal condition will be compared to the GPS and IMU data from the manual drive in inclined condition.

After finishing the manual drive in horizontal condition, a $3.61 \mathrm{~m}$ long steel ramp of $0.175 \mathrm{~m}$ height was placed along the experimental straight path, parallel to the reference lines drawn in the horizontal concrete surface and aligned with the tractor's left side tires. The tractor was driven forwards starting in the flat horizontal portion of the straight path, then it got up into the ramp. Both the front and rear left tires were placed on top of the ramp and the front and rear right tires were on the horizontal surface while the tractor kept traveling forwards, then the tractor got down from the ramp and finished the flat horizontal portion of the straight path. The mean error between the position points recorded along the traveled straight path in horizontal condition and the position points recorded along the traveled path in inclined condition were compared to establish the maximum lateral deviation for this experimental setup. Then, the simulation analysis was performed by using Equation (1) and defining the parameters $(a, b, h)^{T}$ for the traditional correction method and the new correction method; which were applied to the position points $(X, Y, Z)^{T}$ data in combination to the attitude $\left(\varphi, \theta_{p}, \theta_{r}\right)$ data recorded along the traveled inclined path. The parameter's optimization using a simple analytical method was applied to Equation (1) in order to minimize the lateral error and produce the tuned correction method. The simple analytical method consists of selecting the values $(a, b$, $h)^{T}$ from Equation (1) by fine-tuning, as it will be described in Section 3.1. The inclination correction results from each one of the three correction methods were compared to the position points recorded along the traveled straight path by calculating the mean error.

\subsubsection{Automatic guidance experiment}

The second experiment consisted of a test in a fully automatic drive mode in a soil field. The field was not plowed nor tilled, it was bumpy and irregular to make the tractor shake while traveling in order to obtain random inclinations. The purpose of the fully automatic guidance experiment was to verify the accuracy of the three different correction methods from the second experiment in real working conditions. The robot tractor ran in automatic mode using a predesigned navigation map ${ }^{[20]}$ containing straight paths. Each path was around $60 \mathrm{~m}$ in length. No experimental path was traveled more than once; a new different path was traveled during each automatic guidance experiment, thus guaranteeing that the soil was as bumpy as possible, not compacted nor leveled because of the tractor tires' footprint. To verify the accuracy of the three different correction methods, both GPS and IMU data in addition to the robot tractor's steering angle were logged while applying a predefined correction method on each individual experimental path. The robot tractor's steering angle serves as evidence of the automatic guidance system's control action output. In addition, fully automatic guidance experiments without any correction method applied were also performed to obtain the GPS, the IMU and the steering angle data to establish the maximum lateral deviations for this experimental setup and compare them with the results of each one of the three correction methods. The parameter used to establish the maximum lateral deviations was the mean lateral error between the predesigned straight paths and the GPS position points recorded from the automatic drive experiments. In addition, experimental runs were carried out without applying any correction method. This helps to visualize the effect of the position error caused by the tractor's inclinations. The mean error of the steering angle data helped to verify the control action for these lateral deviations. The experimental runs were performed at four different speeds: $0.83 \mathrm{~m} / \mathrm{s}, 1.11 \mathrm{~m} / \mathrm{s}, 1.38 \mathrm{~m} / \mathrm{s}$ and $1.66 \mathrm{~m} / \mathrm{s}$. For each one of the traveling speeds, no correction method and each one of the three correction methods was applied to the robot tractor's automatic guidance system. Therefore, four different traveling speeds by four different correction methods gave as a result of 16 different experimental runs.

\section{Results and discussion}

\subsection{Parameters optimization}

The experimental data from both the GPS and the IMU were processed and plotted using the MATLAB ${ }^{[27]}$ software. Figure 4 shows the GPS position data from the experiment; the position data is displayed in Easting-Northing coordinates. Figure 4a shows the results for a traveling speed of $0.6 \mathrm{~m} / \mathrm{s}$ and Figure $4 \mathrm{~b}$ shows the results for a traveling speed of $3.0 \mathrm{~m} / \mathrm{s}$. The effect of the traveling speed is not evident in the horizontal condition's experiment data, shown in dark gray color. However, the inclined condition's experiment data shown in light gray color is smoother in Figure 4a than in Figure 4b. For the inclined condition experiment, the steel ramp used does not have a completely smooth surface, the steel ramp is like a ladder and thus it provides a bumpy effect that is more notorious at a higher traveling speed. That is the reason why the mean distance between the straight path and the inclined path has a difference of $0.04 \mathrm{~m}$. The mean distance between both paths can also be interpreted as the lateral error of the inclined path. For a traveling speed of $0.60 \mathrm{~m} / \mathrm{s}$, the inclined condition's experiment data indicated in light gray color in Figure 4a is smooth and has a mean distance with respect to the straight path of $0.32 \mathrm{~m}$. However, for a traveling speed of $3.00 \mathrm{~m} / \mathrm{s}$, the inclined condition's experiment data indicated in light gray color in Figure $4 \mathrm{~b}$ is bumpier and exhibits more noticeable peaks and valleys due to the mechanical vibration of the robot tractor's cabin, giving, as a result, a mean distance with respect to the straight path of $0.28 \mathrm{~m}$. Even though, both mean distances are close to each other. Therefore, it is possible to conclude from Figure 4 that both experiments data can be used to apply the position correction method.

Figure 5 shows the IMU attitude data from the experiment; the data is displayed in $\left(^{\circ}\right)$ over time. Figure 5a shows the results for a traveling speed of $0.6 \mathrm{~m} / \mathrm{s}$ and Figure $5 \mathrm{~b}$ shows the results for a traveling speed of $3.0 \mathrm{~m} / \mathrm{s}$. The effect of the traveling speed is evidenced in the time axis; from Figure 5a the experiment took around $47 \mathrm{~s}$ whereas from Figure $5 \mathrm{~b}$ the experiment took around $16 \mathrm{~s}$. The horizontal condition's experiment attitude data shown in dark gray color is almost horizontal for both speeds, which means that the IMU $\left(\varphi, \theta_{p}, \theta_{r}\right)$ measurements are stable despite the mechanical vibration while the robot tractor is traveling in manual mode.

The inclined condition's experiment data shown in light gray color verifies the inclination caused by the steel ramp; which provides 
the tractor a roll angle $\theta_{r}$ of $6.6^{\circ}$. The yaw angle $\varphi$ is almost constant because the robot tractor was driven in a straight path; small oscillations were caused by mechanical vibration. The pitch angle $\theta_{p}$ is exhibited at a peak when the tractor is getting in the ramp because the front left tire is in the ramp, but the rear left tire is in the horizontal concrete surface; giving the tractor a positive pitch.

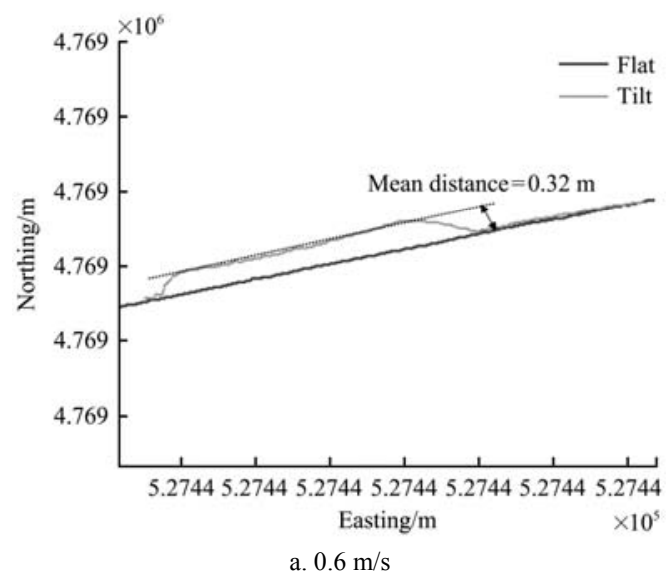

Then, the pitch angle $\theta_{p}$ is horizontal again because both the front and rear left tires are on the ramp. Finally, the pitch angle $\theta_{p}$ exhibits a valley when the tractor is getting off the ramp. It can be concluded from Figure 5 that the IMU $\left(\varphi, \theta_{p}, \theta_{r}\right)$ measurements match the expected behavior for both the horizontal condition experiment and the inclined condition experiment.

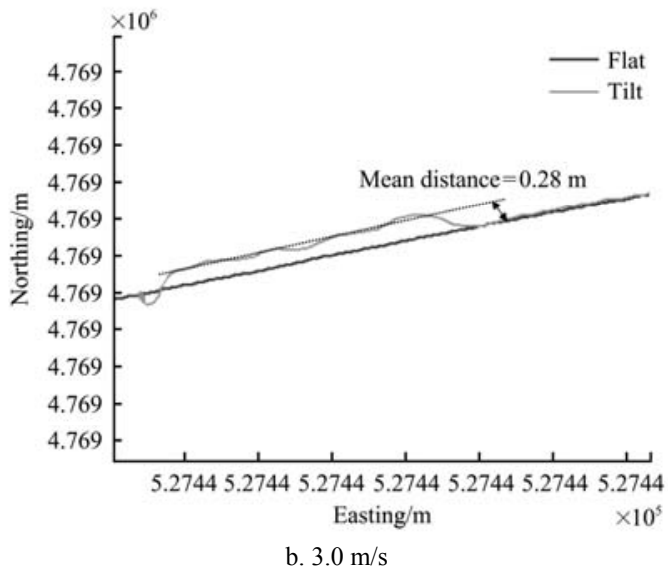

Figure 4 GPS position data from the manual drive experiment
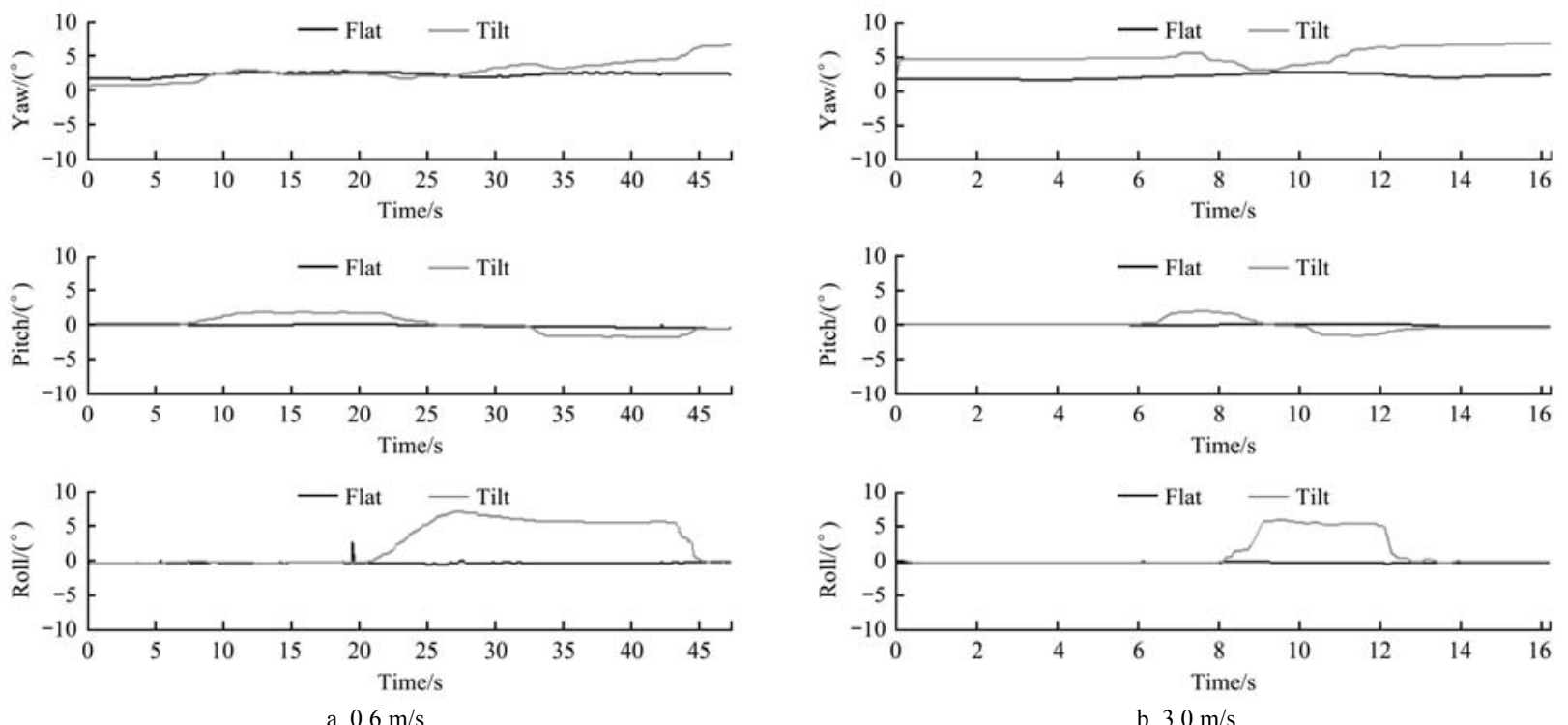

Figure 5 IMU attitude data from the manual drive experiment

To perform the simulation analysis of the correction method, the inclined condition experiment data shown in light gray color in Figure 4 was used as the position input $(X, Y, Z)^{T}$ in Equation (1), in combination with the IMU $\left(\varphi, \theta_{p}, \theta_{r}\right)$ measurements shown in light gray color in Figure 5. The horizontal condition's experiment data shown in dark gray color was used as the reference to estimate the lateral error. The obtained corrected position $\left(X_{a}\right.$, $\left.Y_{a}, Z_{a}\right)^{T}$ was calculated for each method by defining the parameters $(a, b, h)^{T}$. Simply explained, the purpose of each correction method is to obtain a corrected position $\left(X_{a}, Y_{a}, Z_{a}\right)^{T}$ data as close as possible to the flat experiment data. Therefore, hereinafter the inclined condition's experiment data shown in light gray color will be referred to as the raw data. Note that the correction in the $Z$-axis can be neglected because the tractor does not travel in the $Z$-axis, the tractor travels on a horizontal surface changing its $X, Y$ position only.

For the traditional correction method, the parameters $(a, b, h)^{T}$ are defined as the distances from the tractor's $C O G$ to the GPS antenna. Using typical estimations for the tractor's $C O G^{[28]}$, it is possible to assume that the GPS antenna is aligned with the tractor's $C O G$ in the center of the track width ${ }^{[11,29]}$; just like in Figure 3a. Therefore, the lateral distance from the GPS antenna to the tractor's $C O G$ is $a=0.0 \mathrm{~m}$. It is also possible to assume that the GPS antenna is aligned with the tractor's $C O G$ in the center of the wheelbase which gives the longitudinal distance from the GPS antenna to the tractor's $C O G$ as $b=0.0 \mathrm{~m}$. Finally, it is also possible to assume that the GPS antenna's height to the tractor's $C O G$ is $h=2.0 \mathrm{~m}$.

For the new correction method, the parameters $(a, b, h)^{T}$ are defined as the distances from the tractor's pivot point located in the tire to the GPS antenna. The lateral distance from the GPS antenna to the tractor's pivot point as illustrated in Figure $3 \mathrm{~b}$ is half of the track width; therefore, $a=0.0 \mathrm{~m}$. It is also possible to assume $b=0.0 \mathrm{~m}$ because as explained in Section 2.2, the inclination correction of the GPS position error caused by the pitch $\theta_{p}$ is not critical for the performance of a tractor's automated guidance system. Finally, the height from the ground of the GPS' antenna installed in the robot tractor's rooftop was measured 
manually and was found to be $h=2.80 \mathrm{~m}$.

For the parameter optimization that gives, as a result, the tuned correction method, the parameters $(a, b, h)^{T}$ are also defined as the distances from the tractor's pivot point located in the tire to the GPS antenna. The difference is that the values $(a, b, h)^{T}$ are selected instead of measured in order to reduce the lateral error. Therefore, the simple analytical method consists into select the values $(a, b, h)^{T}$ by fine-tuning. The initial $(a, b, h)^{T}$ values were the same as for the new correction method. Using both the Figure 4 GPS position data and the Figure 5 IMU attitude data in Equation (1); the values $(a, b, h)^{T}$ were changed by iterations until the correction method gave as a result of a corrected position $\left(X_{a}, Y_{a}\right.$, $\left.Z_{a}\right)^{T}$ data as close as possible to the horizontal condition's experiment data. Figure 6 shows the error optimization results for $h$; the horizontal axis represents the value of $h$ and the vertical axis represents the mean value of the lateral error. Figure 6a shows the $h$ value iteration for the traveling speed of $0.6 \mathrm{~m} / \mathrm{s}$, and Figure $6 \mathrm{~b}$ shows the results for the traveling speed of $3.0 \mathrm{~m} / \mathrm{s}$. It can be seen in Figure $6 \mathrm{a}$ that for the traveling speed of $0.6 \mathrm{~m} / \mathrm{s}$ the optimal value of $h$ that produces a minimum mean value of the lateral error is $h=2.92 \mathrm{~m}$. It can also be seen in Figure $6 \mathrm{~b}$ that for the traveling speed of $3.0 \mathrm{~m} / \mathrm{s}$ the optimal value of $h$ that produces a minimum mean value of the lateral error is $h=2.98 \mathrm{~m}$. Such difference might be caused by the experimental setup; as it was described and evidenced in Figure 4, there is a bumpy effect that is more notorious at a higher traveling speed. The same approach of value iterations gave as a result the optimal values $a=0.0 \mathrm{~m}$ and $b=0.0 \mathrm{~m}$. The value of $h$ was selected as the average of both $h$ optimal values found for the traveling speed of $0.6 \mathrm{~m} / \mathrm{s}$ and the traveling speed of $3.0 \mathrm{~m} / \mathrm{s}$; i.e. $h=2.95 \mathrm{~m}$.
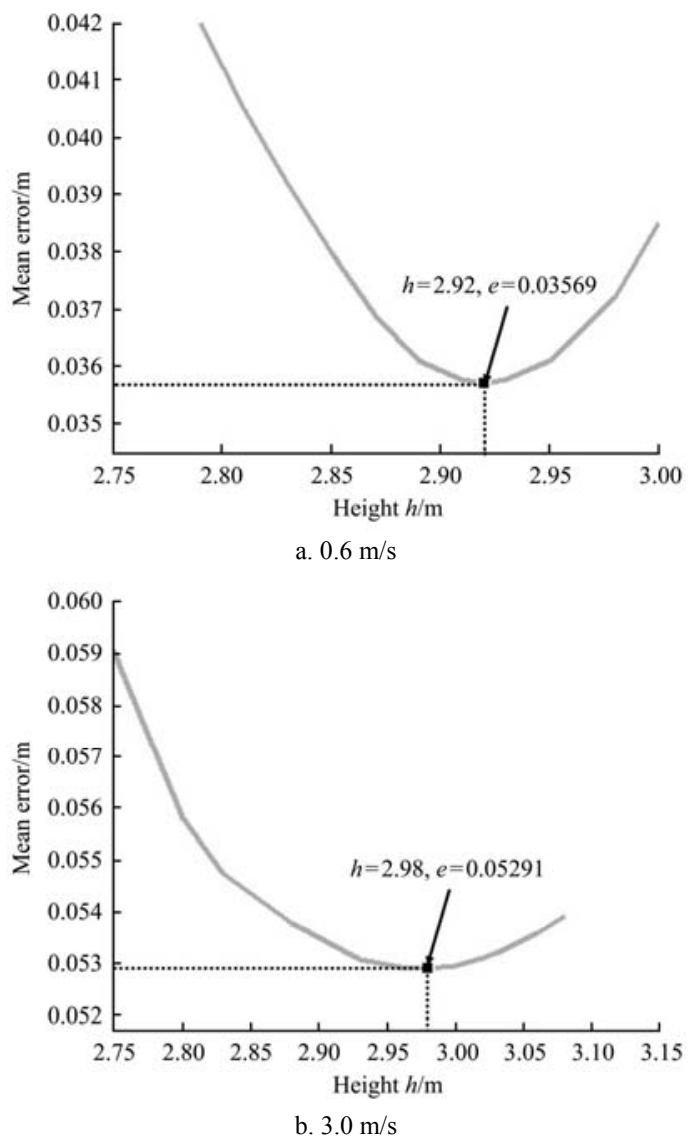

Figure 6 Error optimization results for $h$

Note that it is difficult to explain why the values $(a, b, h)^{T}$ found by the simple analytical method for the tuned correction method differ from the values measured for the new correction method. A mistake in the measurement process of the tractor's track width and height is not likely to happen; although the measurement process is manual, it is simple and easy to verify. Such differences might be caused by factors that are difficult to estimate like the mechanical vibration of the tractor for the different traveling speeds.

Figure 7 shows a summary of the simulation analysis results for all the correction methods. The horizontal axis indicates the path length and the vertical axis represents the lateral deviation; both in meters. Figure 7 a shows the results for a traveling speed of $0.6 \mathrm{~m} / \mathrm{s}$ and Figure $7 \mathrm{~b}$ shows the results for a traveling speed of $3.0 \mathrm{~m} / \mathrm{s}$. The zero value in the vertical axis represents the straight path obtained from the horizontal condition's experiment; as depicted in Figure 4. The inclined condition's experiment data is shown in light gray color; as mentioned before this data will be referred to as the GPS raw data. The mean error with respect to the horizontal condition's experiment data; i.e. the zero value in the vertical axis, along all the traveled path was calculated to estimate the accuracy. Please note that this means error is different from the mean distance shown in Figure 4; the mean distance was not calculated along all the traveled path; it was calculated only for the inclined portion of the path to knowing the GPS antenna maximum deviation for this experimental setup. Figure 7 a shows that the GPS raw data have a mean error of $0.21 \mathrm{~m}$ for a traveling speed of $0.6 \mathrm{~m} / \mathrm{s}$, whereas Figure $7 \mathrm{~b}$ shows that the GPS raw data have a mean error of $0.17 \mathrm{~m}$ for a traveling speed of $3.0 \mathrm{~m} / \mathrm{s}$. Although the mean error is smaller for the experiment at $3.0 \mathrm{~m} / \mathrm{s}$, the data displays a bigger oscillation caused by the bumpy effect of the steel ramp. The final part of the data from Figure $7 \mathrm{~b}$ also has a bigger lateral error caused by the whip effect of the cabin when the tractor is getting off the ramp. Even though, the data from Figure $7 \mathrm{~b}$ is still useful for simulation purposes because in real working conditions the tractor will travel on a bumpy soil surface.

The result of the traditional correction method is indicated by the dark gray dotted line in Figure 7. Figure 7a shows that the traditional correction method result has a mean error of $0.086 \mathrm{~m}$ for a traveling speed of $0.6 \mathrm{~m} / \mathrm{s}$, whereas Figure $7 \mathrm{~b}$ shows that the traditional correction method result has a mean error of $0.064 \mathrm{~m}$ for a traveling speed of $3.0 \mathrm{~m} / \mathrm{s}$. Such results are not bad, considering the assumption of the parameters $(a, b, h)^{T}$ respect to the tractor's COG. This gives an idea of why this method is commonly used $^{[18,21]}$ with satisfactory results. Even though, it is possible to improve this result.

The result of the new correction method is indicated by the light gray dotted line in Figure 7. Figure 7a shows that the new correction method result has a mean error of $0.041 \mathrm{~m}$ for a traveling speed of $0.6 \mathrm{~m} / \mathrm{s}$, whereas Figure $7 \mathrm{~b}$ shows that the new correction method result has a mean error of $0.056 \mathrm{~m}$ for a traveling speed of $3.0 \mathrm{~m} / \mathrm{s}$. Please consider that although the mean error reduction might not seem significant for a traveling speed of $3.0 \mathrm{~m} / \mathrm{s}$, considering that the definition and measurement of the parameters $(a, b, h)^{T}$ for this new correction method is simpler than the assumption of the parameters $(a, b, h)^{T}$ respect to the tractor's $C O G$ for the traditional correction method, this result proves that the new correction method is not only more practical and easy to implement but also is more precise.

The result of the tuned correction method is indicated by the black dotted line in Figure 7. Figure 7a shows that the tuned correction method result has a mean error of $0.036 \mathrm{~m}$ for a traveling speed of $0.6 \mathrm{~m} / \mathrm{s}$, whereas Figure $7 \mathrm{~b}$ shows that the tuned 
correction method result has a mean error of $0.053 \mathrm{~m}$ for a traveling speed of $3.0 \mathrm{~m} / \mathrm{s}$. The improvement with respect to the new correction method is not too big. However, considering that the parameters $(a, b, h)^{T}$ for the tuned correction method were found analytically with the purpose of obtaining the smallest error possible, it is possible to conclude that the measurements of the parameters $(a, b, h)^{T}$ for the new correction method are not far away from being optimal.
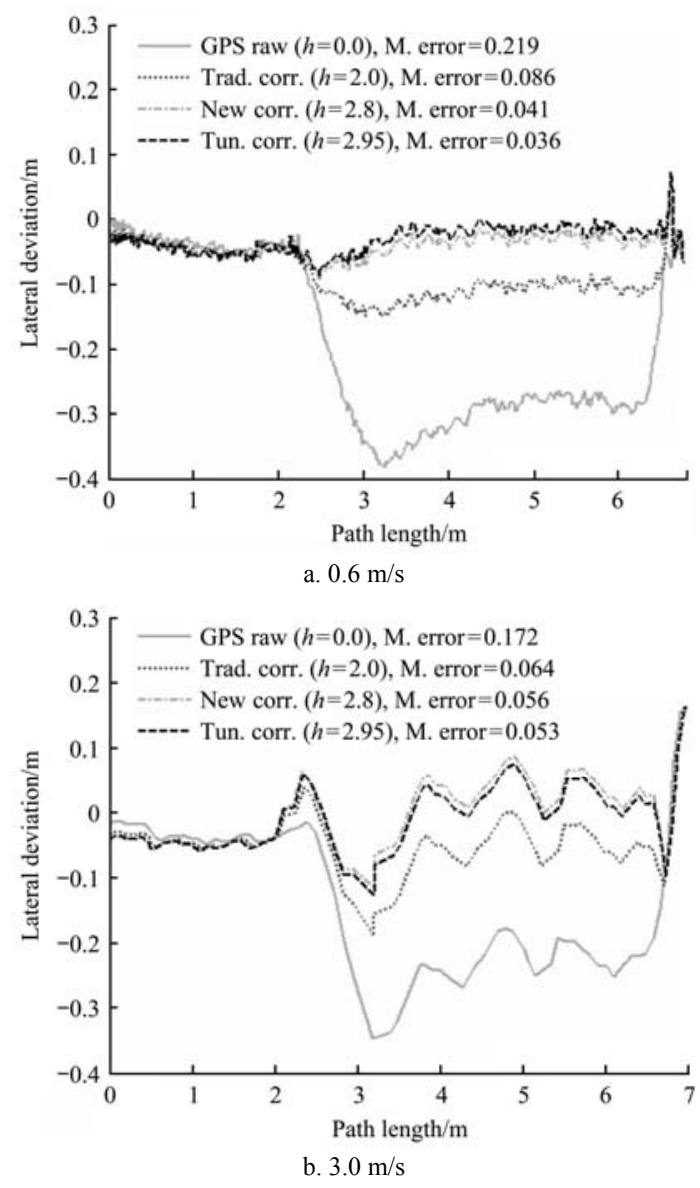

Figure 7 Parameter optimization results for $h$

The error reduction might seem obvious by looking at the trends from Figure 7, but it is easier to compare the data numerically. Table 1 shows a summary of the experimental results from Figure 7 for clarification purposes. The name of the correction method is listed on the left followed by its respective parameter $h$. The mean error results for both traveling speeds are displayed in meters. It is easy to see in Table 1 the gradual reduction of the mean error data for each correction method from top to bottom of the table for the traveling speed of $0.6 \mathrm{~m} / \mathrm{s}$. The percentage of error reduction for each correction method is listed on the right side of the mean error in the table; this percentage of error reduction was calculated from each correction method with respect to the GPS raw data. It is possible to see the gradual reduction of the mean error data from top to bottom in the table. For the traditional correction method, the mean error with respect to the GPS raw data was reduced $60.7 \%$ for a traveling speed of $0.6 \mathrm{~m} / \mathrm{s}$, and $62.7 \%$ for a traveling speed of $3.0 \mathrm{~m} / \mathrm{s}$. For the new correction method, the mean error with respect to the GPS raw data was reduced $81.2 \%$ for a traveling speed of $0.6 \mathrm{~m} / \mathrm{s}$, and around $67.4 \%$ for a traveling speed of $3.0 \mathrm{~m} / \mathrm{s}$. This means that the new correction method is around $21.0 \%$ more accurate in comparison to the traditional correction method for low speeds. For the tuned correction method, the mean error with respect to the GPS raw data was reduced $83.5 \%$ for a traveling speed of $0.6 \mathrm{~m} / \mathrm{s}$, and $69.1 \%$ for a traveling speed of $3.0 \mathrm{~m} / \mathrm{s}$. This means that the tuned correction method obtained by parameter optimization is around $23 \%$ more accurate in comparison to the traditional correction method for low speeds. In the case of a traveling speed of $3.0 \mathrm{~m} / \mathrm{s}$, there is a slight improvement of around 5\%-7\% for both the new correction method and the tuned correction method in comparison to the traditional correction method. Although the mean error reduction is not as big for the traveling speed of $0.6 \mathrm{~m} / \mathrm{s}$, the mean error reduction tendency can also be observed in Table 1. This means that despite the traveling speed of $3.0 \mathrm{~m} / \mathrm{s}$ being way beyond the typical operating speed for a tractor in working conditions ${ }^{[16]}$, the correction method is still effective. In conclusion, the error reduction provided by the new correction method and the tuned correction method was significant for a travelling speed of $0.6 \mathrm{~m} / \mathrm{s}$. For a traveling speed of $3.0 \mathrm{~m} / \mathrm{s}$, the error reduction is also evidenced but is less significant.

Table 1 Summary of the parameter optimization results

\begin{tabular}{lcccccc}
\hline & & \multicolumn{2}{c}{$v=0.6 \mathrm{~m} / \mathrm{s}$} & & \multicolumn{2}{c}{$v=3.0 \mathrm{~m} / \mathrm{s}$} \\
\cline { 3 - 5 } \cline { 5 - 6 } & $h / \mathrm{m}$ & $\begin{array}{c}\text { Mean } \\
\text { error/m }\end{array}$ & $\begin{array}{c}\text { Mean error } \\
\text { reduction/\% }\end{array}$ & $\begin{array}{c}\text { Mean } \\
\text { error/m }\end{array}$ & $\begin{array}{c}\text { Mean error } \\
\text { reduction/\% }\end{array}$ \\
\hline GPS raw & 0.00 & 0.219 & - & 0.172 & - \\
Traditional method & 2.00 & 0.086 & 60.73 & 0.064 & 62.79 \\
New method & 2.80 & 0.041 & 81.27 & 0.056 & 67.44 \\
Tuned method & 2.95 & 0.036 & 83.56 & 0.053 & 69.18 \\
\hline
\end{tabular}

As a final remark, for the parameter's optimization experiment the ground conditions are too different from the real working conditions in the field. Therefore, these results do not guarantee that the performance of the correction methods is analogous to a bumpy soil condition. It is still necessary to verify experimentally the accuracy of the correction methods under real working conditions.

\subsection{Automatic guidance experiment}

Figure 8 shows a summary of the results for the automatic guidance experiment for a traveling speed of $0.83 \mathrm{~m} / \mathrm{s}$. The GPS raw data is shown in light gray color in all the plots in Figure 8; it corresponds to the experimental paths in which no correction method is applied. Analogous to Figure 7, in Figure 8 the results of the traditional correction method are indicated by the dark gray dotted line, the results of the new correction method are indicated by the light gray dotted line and the results of the tuned correction method are indicated by the black dotted line. Similar results were obtained for the traveling speeds of $1.11 \mathrm{~m} / \mathrm{s}, 1.38 \mathrm{~m} / \mathrm{s}$, and $1.66 \mathrm{~m} / \mathrm{s}$; but the plots are not included because of space considerations. Instead, the data results are discussed below and are summarized in Table 2.

Figure 8a summarizes the lateral error results for a traveling speed of $0.83 \mathrm{~m} / \mathrm{s}$. The vertical axis corresponds to the lateral error in meters and the horizontal axis corresponds to the path length. The zero value in the vertical axis represents the target path traveled. It can be visually verified that the lateral errors have an initial offset that is not consistent. Such an initial offset was caused by the initial position of the robot tractor with respect to the starting point of the navigation map used in the automatic guidance system. Although the robot tractor traveled in automatic mode, parking it in the initial position was a manual process aided by using ground landmarks. Please note that the initial offsets observed in Figure 8a do not exceed $0.30 \mathrm{~m}$ in the worst case, and are usually smaller than $0.20 \mathrm{~m}$. This initial offset does not affect significantly the performance of the inclination correction method 
because the navigation controller in the automatic guidance system takes the robot tractor as close as possible to the target path as fast as possible. For example, Figure 8a notes that the biggest initial offset of $0.30 \mathrm{~m}$ corresponds to the new correction method's experimental path; even so, the new correction method performs better than the traditional correction method giving as a result of smaller oscillation with respect to the zero value in the vertical axis.

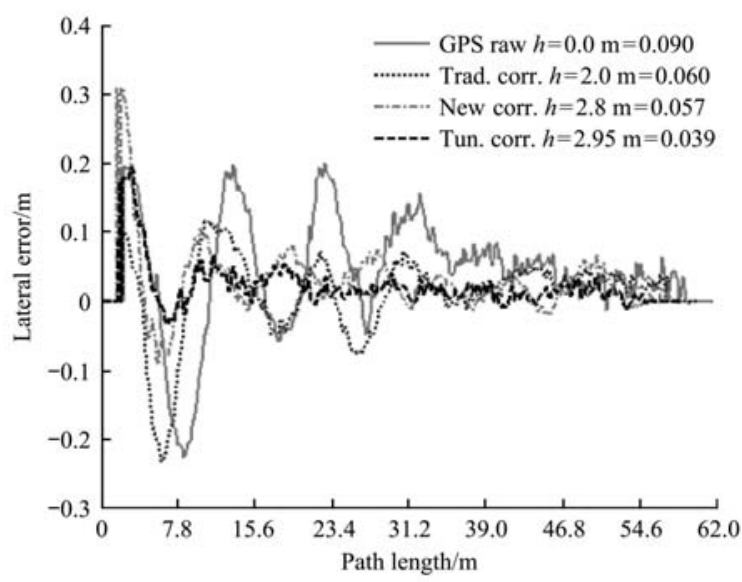

a. Lateral error $(v=0.83 \mathrm{~m} / \mathrm{s})$

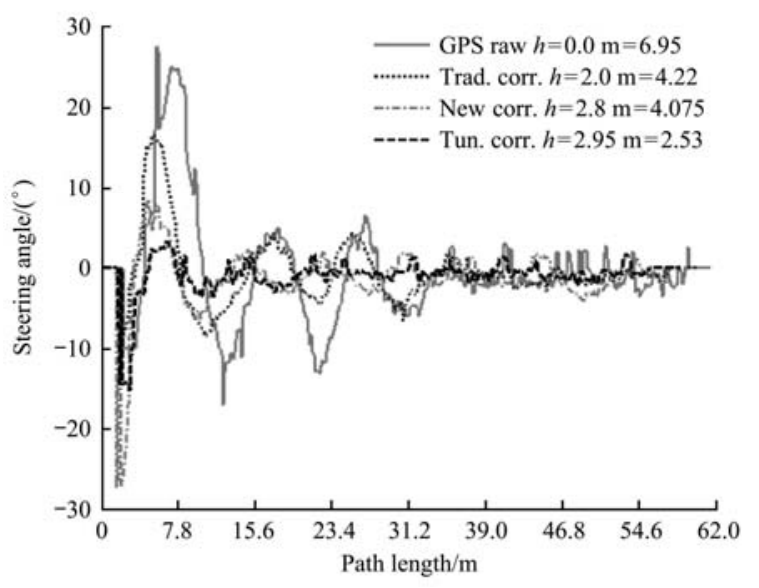

b. Steering angle $(v=0.83 \mathrm{~m} / \mathrm{s})$

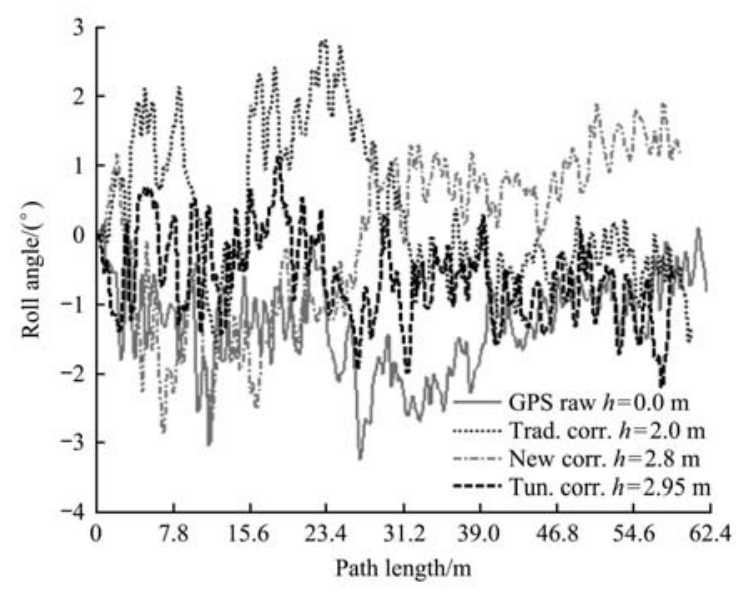

c. Roll angle $(v=0.83 \mathrm{~m} / \mathrm{s})$

Figure 8 Automatic guidance experiment results

The automatic guidance experiment results summarized in Figure 8 a display similar behavior to the results summarized in Figure 7 for the parameters optimization experiment. The RMSE of the lateral error with respect to the zero value in the vertical axis; i.e. the target path traveled, was calculated to estimate the accuracy of each correction method. In Figure 8a, the GPS raw data shows the highest RMSE of the lateral error (RMSE $=0.090 \mathrm{~m}$ ), and such error reduces gradually by applying the traditional correction method $(\mathrm{RMSE}=0.060 \mathrm{~m})$, the new correction method $(\mathrm{RMSE}=$ $0.057 \mathrm{~m}$ ) and the tuned correction method (RMSE $=0.039 \mathrm{~m})$. The same behavior was observed for a traveling speed of $1.11 \mathrm{~m} / \mathrm{s}$; the GPS raw data calculated RMSE of the lateral error is the highest (RMSE $=0.060 \mathrm{~m}$ ), and such error reduces gradually by applying the traditional correction method $(\mathrm{RMSE}=0.043 \mathrm{~m}$ ), the new correction method $(\mathrm{RMSE}=0.041 \mathrm{~m})$ and the tuned correction method $(\mathrm{RMSE}=0.039 \mathrm{~m})$. These results verify the results obtained in the parameters optimization experiment.

However, for both traveling speeds of $1.38 \mathrm{~m} / \mathrm{s}$ and of $1.66 \mathrm{~m} / \mathrm{s}$ the performance of all correction methods was approximately the same in comparison to the GPS raw data. In all cases, the data is roughly the same. The inclination correction effect was not visible. For a traveling speed of $1.38 \mathrm{~m} / \mathrm{s}$ the GPS raw data calculated RMSE of the lateral error is big (RMSE $=0.075 \mathrm{~m}$ ), and such error is roughly the same when applying the traditional correction method $(\mathrm{RMSE}=0.054 \mathrm{~m})$, the new correction method $(\mathrm{RMSE}=0.049 \mathrm{~m})$ and the tuned correction method (RMSE= $0.045 \mathrm{~m}$ ). Similarly, for a traveling speed of $1.66 \mathrm{~m} / \mathrm{s}$ the GPS raw data calculated RMSE of the lateral error is high (RMSE= $0.049 \mathrm{~m}$ ), and such error remains the same when applying the traditional correction method (RMSE $=0.049 \mathrm{~m}$ ), the new correction method (RMSE $=0.044 \mathrm{~m}$ ) and the tuned correction method $(\mathrm{RMSE}=0.046 \mathrm{~m})$. Note that from the calculated RMSE of the lateral error for a traveling speed of $1.66 \mathrm{~m} / \mathrm{s}$ the traditional correction method has the same lateral error compared to the GPS raw data; this means that for this experimental setup at a higher speed, the traditional method correction effect is not significant. In conclusion, if the traveling speed increases, the performance of all the correction methods degrades. These results make sense for a couple of factors. First, the GPS antenna measurements' oscillations (caused by the bumpy effect of the surface where the tractor is moving on) increase if the traveling speed increases, creating a whip effect of the cabin when the tractor is getting on and off a slope in the soil. This effect was already evidenced in Figure 7b). Second, the sensors measurement and the automatic guidance system's controller have an update rate of $10 \mathrm{~Hz}$, causing the robot tractor's control action response to be off-time respect to a faster change over time in its position and inclination when traveling at a higher speed. This means that the robot tractor's real position and attitude change a little faster than what the robot tractor can measure. Initially, this is not a big issue because a traveling speed of $1.66 \mathrm{~m} / \mathrm{s}(6 \mathrm{~km} / \mathrm{h})$ is a little above the typical operating speeds for a tractor pulling an implement ${ }^{[16]}$.

Figure $8 \mathrm{~b}$ corresponds to the steering angle results for a traveling speed of $0.83 \mathrm{~m} / \mathrm{s}$. The vertical axis corresponds to the steering angle in $\left(^{\circ}\right)$, and the horizontal axis corresponds to the path length. The zero value in the vertical axis represents the condition in which the robot tractor travels perfectly aligned to the target path and therefore no steering control action is needed. Such a condition is not possible, but it can be used as a steering reference. The robot tractor's steering angle serves as evidence of the automatic guidance system's control action output according to the inclination correction method applied. This means that if no correction method is applied, the GPS antenna position measurements have a bigger lateral error and therefore the robot tractor needs a bigger steering maneuver to try to recover its position with respect to the target path. Similarly, if a correction method is applied then the GPS antenna position measurements 
have a smaller lateral error; therefore, the robot tractor's steering maneuver to position itself into the target path is smaller. Logically, the robot tractor's initial position causes an initial offset that affects directly the steering maneuver; but as explained in Figure $8 \mathrm{a}$ the initial offsets do not exceed $0.30 \mathrm{~m}$ in the worst case and are usually smaller than $0.20 \mathrm{~m}$ so they should not affect the steering maneuver significantly. This statement is verified in Figure $8 \mathrm{~b}$, the initial steering maneuver is not too pronounced $\left(\sim 10^{\circ}\right)$ for the first $10 \mathrm{~m}$ of the traveled path but after that, it stabilizes for the rest of the traveled path. In conclusion, the initial offset does not affect significantly the steering control action because the initial steering maneuver is smaller than $15^{\circ}$ and it stabilizes quickly.

The RMSE of the steering angle with respect to the zero value in the vertical axis was calculated to verify the accuracy of each correction method. In Figure $8 \mathrm{~b}$ for a traveling speed of $0.83 \mathrm{~m} / \mathrm{s}$; the GPS raw data shows the highest RMSE of the steering angle $\left(\mathrm{RMSE}=6.953^{\circ}\right)$, and such RMSE of the steering angle reduces gradually by applying the traditional correction method $\left(\mathrm{RMSE}=4.228^{\circ}\right)$, the new correction method $\left(\mathrm{RMSE}=4.075^{\circ}\right)$ and the tuned correction method $\left(\mathrm{RMSE}=2.534^{\circ}\right)$. The same behavior was verified for a traveling speed of $1.11 \mathrm{~m} / \mathrm{s}$; the GPS raw data has the highest calculated RMSE of the steering angle $\left(\mathrm{RMSE}=4.461^{\circ}\right)$, and such RMSE of the steering angle reduces gradually by applying the traditional correction method $\left(\mathrm{RMSE}=2.924^{\circ}\right)$, the new correction method $\left(\mathrm{RMSE}=2.673^{\circ}\right)$ and the tuned correction method $\left(\mathrm{RMSE}=2.546^{\circ}\right)$. These results verify the results obtained in the parameters optimization experiment.

Again, for both traveling speeds of $1.38 \mathrm{~m} / \mathrm{s}$ and of $1.66 \mathrm{~m} / \mathrm{s}$ the control action indicated by the steering angle is roughly the same in comparison to the GPS raw data. For a traveling speed of $1.38 \mathrm{~m} / \mathrm{s}$ the GPS raw data calculated RMSE of the steering angle is big $\left(\mathrm{RMSE}=4.738^{\circ}\right.$ ), and such RMSE of the steering angle is approximately the same when applying the traditional correction method $\left(\mathrm{RMSE}=3.679^{\circ}\right)$, the new correction method $(\mathrm{RMSE}=$ $3.547^{\circ}$ ) and the tuned correction method (RMSE $=3.032^{\circ}$ ). Similarly, for a traveling speed of $1.38 \mathrm{~m} / \mathrm{s}$ the GPS raw data calculated RMSE of the steering angle is big $\left(\operatorname{RMSE}=3.298^{\circ}\right)$, and such RMSE of the steering angle remains almost the same when applying the traditional correction method $\left(\mathrm{RMSE}=3.412^{\circ}\right)$, the new correction method $\left(\mathrm{RMSE}=2.874^{\circ}\right)$ and the tuned correction method $\left(\mathrm{RMSE}=2.766^{\circ}\right)$. In a conclusion, these results are just as expected because they reflect the behavior of the GPS antenna position measurements explained in Figure 8a. This means that higher position lateral errors caused by bigger inclinations of the GPS antenna cause bigger control actions in the steering angle. As evidenced in Figure $8 \mathrm{~b}$ smaller position lateral errors caused by better correction of the inclinations of the GPS antenna, can cause smaller control actions in the steering angle.

Figure $8 \mathrm{c}$ corresponds to the roll angle $\theta_{r}$ recorded for the four paths with a traveling speed of $0.83 \mathrm{~m} / \mathrm{s}$. The vertical axis corresponds to the roll angle $\theta_{r}$ in $\left(^{\circ}\right)$ and the horizontal axis corresponds to the path length. The roll angle $\theta_{r}$ serves as evidence of the inclinations caused by the bumpy soil on which the robot tractor traveled. If the soil conditions are roughly the same, the roll angle $\theta_{r}$ should be within the same range for all the 16 experimental paths. If one experimental path would be flatter and smoother than the others, then the roll angle $\theta_{r}$ would be almost zero, smooth and horizontal. Similarly, if one experimental path would be bumpier and more potholed than the others, then the roll angle $\theta_{r}$ would have a wider range and abrupter oscillations. This means that the differences evidenced for each correction method results from Figure $8 \mathrm{a}$ and Figure $8 \mathrm{~b}$ are a product of the accuracy of the correction method applied on each experimental run and not a product of mere coincidence or more favorable soil conditions. The oscillations evidenced within the first $10 \mathrm{~m}$ of the path length for lateral error results summarized in Figure $8 \mathrm{a}$ and the steering angle results summarized in Figure $8 \mathrm{~b}$ do not correspond to the inclination effect evidenced by the roll angle $\theta_{r}$ measurements summarized in Figure 8c. These oscillations correspond to the offset caused by the initial position of the robot tractor with respect to the starting navigation point. Note that the roll angle $\theta_{r}$ measurements summarized in Figure 8c are within a range of $7.0^{\circ}$ maximum and all the measurements display roughly the same behavior. Please remember that from the parameter's optimization section the inclined condition's experiment verified the inclination caused by the steel ramp; which provided the tractor a roll angle $\theta_{r}$ of $6.6^{\circ}$. This value is not far from the range for the roll angle $\theta_{r}$ measurements summarized in Figure $8 \mathrm{c}$. Similar to Figure 5, the calculation of the RMSE of the roll angle $\theta_{r}$ is omitted in Figure $8 \mathrm{c}$ because it does not provide useful information about the performance or effectiveness of each inclination correction method, it provides a notion of the similarities of the soil conditions. That is the reason why the roll angle $\theta_{r}$ measurements summarized in Figure 8c look similar despite the correction method label. These are attitude data measurements; no further processing action is performed to modify them. This means that the roll angle $\theta_{r}$ measurements summarized in Figure $8 \mathrm{c}$ are used in combination with Equation (1) and Equation (2) as input data to correct the GPS position measurements giving, as a result, the plots in Figure 8a. Therefore, it is possible to affirm that the soil conditions were roughly the same for the four paths with a traveling speed of $0.83 \mathrm{~m} / \mathrm{s}$. Similar roll angle $\theta_{r}$ measurements were obtained for all the other 12 experimental paths for the traveling speeds of $1.11 \mathrm{~m} / \mathrm{s}, 1.38 \mathrm{~m} / \mathrm{s}$ and $1.66 \mathrm{~m} / \mathrm{s}$. In conclusion, the soil conditions were similar for all the 16 experimental paths and they did not interfere with the inclination correction methods' result.

For the automatic guidance experiment, the error reduction is difficult to see from the trends in Figure 8 and the discussion above, so it is easier to compare the data numerically. Table 2 shows a summary of the experimental results for the four different travel speeds tested. For each correction method, the RMSE values of the lateral error and the steering angle were calculated with respect to the target path and the zero steering value respectively. The percentage of the lateral error RMSE reduction was calculated with respect to the GPS raw data.

For the traditional correction method, the lateral error RMSE with respect to the GPS raw data was reduced $33.33 \%$ for a traveling speed of $0.83 \mathrm{~m} / \mathrm{s}$ and $28.33 \%$ for a traveling speed of $1.11 \mathrm{~m} / \mathrm{s}$. For a traveling speed of $1.38 \mathrm{~m} / \mathrm{s}$ the lateral error RMSE was reduced $28.00 \%$ but it was not reduced $(0.00 \%)$ for a traveling speed of $1.66 \mathrm{~m} / \mathrm{s}$; which means that the traditional correction method does not provide any impact in the lateral error RMSE reduction at higher speeds.

For the new correction method, the lateral error RMSE with respect to the GPS raw data was reduced $36.66 \%$ for a traveling speed of $0.83 \mathrm{~m} / \mathrm{s}, 31.66 \%$ for a traveling speed of $1.11 \mathrm{~m} / \mathrm{s}$ and $34.66 \%$ for a traveling speed of $1.38 \mathrm{~m} / \mathrm{s}$. However, it was reduced by just $10.20 \%$ for a traveling speed of $1.66 \mathrm{~m} / \mathrm{s}$. This means that the new correction method had an RMSE reduction 
percentage up to $10 \%$ better than the traditional correction method.

Similarly, for the tuned correction method the lateral error RMSE with respect to the GPS raw data was reduced $56.66 \%$ for a traveling speed of $0.83 \mathrm{~m} / \mathrm{s}, 35.00 \%$ for a traveling speed of $1.11 \mathrm{~m} / \mathrm{s}$ and $40.00 \%$ for a traveling speed of $1.38 \mathrm{~m} / \mathrm{s}$. Finally, it was reduced only by $6.12 \%$ for a traveling speed of $1.66 \mathrm{~m} / \mathrm{s}$. This means that the tuned correction method had an RMSE reduction percentage up to $23 \%$ better than the traditional correction method.

In conclusion, in comparison to the traditional correction method results, the error reduction provided by the new correction method and the tuned correction method was significant for a travelling speed of $0.83 \mathrm{~m} / \mathrm{s}$ and $1.11 \mathrm{~m} / \mathrm{s}$. For a travelling speed of $1.38 \mathrm{~m} / \mathrm{s}$ and $1.66 \mathrm{~m} / \mathrm{s}$ the GPS raw data is too similar to the results of the traditional correction method, the new correction method and the tuned correction method; giving, as a result, an almost null error reduction.

Similar research ${ }^{[18]}$ using the traditional correction method gave, as a result, a lateral error of less than about $0.2 \mathrm{~m}$ and an RMSE of $0.081 \mathrm{~m}$, which is close to the values in Table 2 for a travelling speed of $0.83 \mathrm{~m} / \mathrm{s}$. Please note that it might be difficult to compare results summarized in Table 2 directly with the results obtained in Reference [18] because there is no guarantee that the bumpy soil conditions and the experimental setup were the same. The performance of the traditional correction method seems to be similar. As a final remark, since the automatic guidance experimental conditions were the same as the real working conditions in the field; this approach helped to verify experimentally the error reduction of each one of the correction methods. The data from Figure 8 and the summary from Table 2 helped to understand that there is traveling speed dependency that affects the performance of all the correction methods. Even though, these results are consistent with the results from the parameters optimization experiment, and it was possible to verify that the new correction method and the tuned correction method have an overall lateral error reduction in comparison with the traditional correction method.

Table 2 Summary of the automatic drive experiment results

\begin{tabular}{|c|c|c|c|c|c|c|c|c|c|c|c|c|}
\hline & \multicolumn{3}{|c|}{$v=0.83 \mathrm{~m} / \mathrm{s}$} & \multicolumn{3}{|c|}{$v=1.11 \mathrm{~m} / \mathrm{s}$} & \multicolumn{3}{|c|}{$v=1.38 \mathrm{~m} / \mathrm{s}$} & \multicolumn{3}{|c|}{$v=1.66 \mathrm{~m} / \mathrm{s}$} \\
\hline & $\begin{array}{c}\text { Lateral } \\
\text { error } \\
\text { RMSE/m }\end{array}$ & $\begin{array}{l}\text { Steering } \\
\text { angle } \\
\text { RMSE/( }\left(^{\circ}\right)\end{array}$ & $\begin{array}{c}\text { Lateral } \\
\text { error } \\
\text { reduction/\% }\end{array}$ & $\begin{array}{c}\text { Lateral } \\
\text { error } \\
\text { RMSE/m }\end{array}$ & $\begin{array}{l}\text { Steering } \\
\text { angle } \\
\text { RMSE/( }\left(^{\circ}\right)\end{array}$ & $\begin{array}{c}\text { Lateral } \\
\text { error } \\
\text { reduction/\% }\end{array}$ & $\begin{array}{c}\text { Lateral } \\
\text { error } \\
\text { RMSE/m }\end{array}$ & $\begin{array}{c}\text { Steering } \\
\text { angle } \\
\text { RMSE }^{\circ}\left({ }^{\circ}\right)\end{array}$ & $\begin{array}{c}\text { Lateral } \\
\text { error } \\
\text { reduction/\% }\end{array}$ & $\begin{array}{c}\text { Lateral } \\
\text { error } \\
\text { RMSE/m }\end{array}$ & $\begin{array}{c}\text { Steering } \\
\text { angle } \\
\mathrm{RMSE}^{\circ}\left({ }^{\circ}\right)\end{array}$ & $\begin{array}{c}\text { Lateral } \\
\text { error } \\
\text { reduction/\% }\end{array}$ \\
\hline GPS raw & 0.090 & 6.953 & - & 0.060 & 4.461 & - & 0.075 & 4.738 & - & 0.049 & 3.298 & - \\
\hline Traditional method & 0.060 & 4.228 & 33.33 & 0.043 & 2.924 & 28.33 & 0.054 & 3.679 & 28.00 & 0.049 & 3.412 & 0.00 \\
\hline New method & 0.057 & 4.075 & 36.66 & 0.041 & 2.673 & 31.66 & 0.049 & 3.547 & 34.66 & 0.044 & 2.874 & 10.20 \\
\hline Tuned method & 0.039 & 2.534 & 56.66 & 0.039 & 2.546 & 35.00 & 0.045 & 3.032 & 40.00 & 0.046 & 2.766 & 6.12 \\
\hline
\end{tabular}

\section{Conclusions}

This research presented a new inclination correction method and a tuned correction method applied to the guidance system of an agricultural vehicle. The traditional method was described in order to identify the possibility of improving it. The considerations required to apply the new method were described according to the geometry of a robot tractor. The fact that it is not necessary to calculate the tractor's center of gravity constitutes the practical advantage of the new correction method.

The parameters optimization experiment helped to verify the considerations required to apply the new method and the effect of the roll angle on the position error when the tractor was traveling at an approximately constant speed. It mainly helped to produce the tuned correction method; by means of parameter optimization using a simple analytical method applied to the correction equation, in order to minimize the lateral error. The results from each of the three correction methods were compared. The new correction method produced a mean error reduction percentage up to $21 \%$ better than the traditional correction method. The tuned correction method produced a mean error reduction percentage up to $23 \%$ better than the traditional correction method when the vehicle was travelling on a flat surface.

The automatic guidance experiment helped to verify the results from the parameters optimization experiment under real working conditions. The results were verified by calculating the RMSE of the lateral error and such error reduction percentage. The new correction method produced a mean error reduction percentage up to $7 \%$ better than the traditional correction method. The tuned correction method produced a mean error reduction percentage up to $23 \%$ better than the traditional correction method when the vehicle was travelling on a bumpy soil surface. The performance of all the correction method degrades as the travelling speed increases. Even though, the new inclination correction method and the tuned correction method introduced in this research show an overall improvement compared to the traditional correction method.

\section{[References]}

[1] Coulmas F. Population decline and ageing in Japan - The social consequences. New York: Ed. Routledge, 2007; 168p.

[2] Kondo N, Monta M, Noguchi N. Agricultural robots: mechanisms and practice. Kyoto: University Press and Trans Pacific Press, 2011; 348p.

[3] Zhang Z, Noguchi N, Ishii K. Development of a robot combine harvester. Journal of the Japanese Society of Agricultural Machinery and Food Engineers, 2015; 77(1): 45-50.

[4] Takai R, Yang L, Noguchi N. Development of crawler-type robot tractor based on GNSS and IMU. IFAC Proceedings Volumes, 2013; 46(4): 95-98.

[5] Lahtinen J. How mems sensors linked to GPS systems boost crop yields. eeNews Embedded. 2015. Available: https://www.eenewsembedded.com/blog/how-mems-sensors-linked-gps-sy stems-boost-crop-yields. Accessed on [2020-06-20].

[6] Ryu J, Rossetter E J, Gerdes J C. Vehicle sideslip and roll parameter estimation using GPS. AVEC 2002 6th Int. Symposium on Advanced Vehicle Control, Hiroshima, Japan, 2002; pp.373-380.

[7] Bae H S, Ryu J, Gerdes C. Road grade and vehicle parameter estimation for longitudinal control using GPS. Proceedings of the IEEE Conference on Intelligent Transportation Systems, August 2001. Oakland: IEEE. 2001; pp.166-171.

[8] Germann S T, Isermann R. Determination of the centre of gravity Height of a vehicle with parameter estimation. IFAC Proceedings Volumes, 1994; 27(8): 563-568.

[9] Ospina R, Noguchi N. Determination of tire dynamic properties: application to an agricultural vehicle. EAEF, 2016; 9: 123-130.

[10] Wong J Y. Theory of ground vehicles, Second Ed. New York: John Wiley \& Sons, Inc. 1993; 435p.

[11] Liljedahl J B, Turnquist P K, Smith D W, Hoki M. Tractors and their 
power units, fourth ed. New York: Van Nostrand Reinhold, 1989; pp.303-306.

[12] Kim Y, Noh J, Shin S, Kim B, Hong S. Improved method for determining the height of center of gravity of agricultural tractors. Journal of Biosystems Engineering, 2016; 41(3): 170-176.

[13] Al-Rawashdeh Y M, Elshafei M, Al-Malki M F. In-flight estimation of center of gravity position using all-accelerometers. Sensors, 2014; 14: 17567-17585.

[14] Deng Z, Chu D, Tian F, He Y, Wu C, Hu Z, et al. Online estimation for vehicle center of gravity height based on unscented Kalman filter. 2017 4th International Conference on Transportation Information and Safety (ICTIS), Banff: IEEE, 2017; pp.33-36.

[15] Claar P, Buchele W, Marley S, Sheth P. Agricultural tractor chassis suspension system for improved ride comfort. SAE Technical Paper, 1980; 801020. doi: 10.4271/801020.

[16] Ruitao G, Yang W, Zhou Y, Hong J, Zhixiang L, Jian S. Tractor driving seat suspension system research status and strategies in China: A review. IFAC-PapersOnLine, 2018; 51(17): 576-581.

[17] Han X, Kim H J, Jeon C W, Moon H C, Kim J H, Yi S Y. Application of a 3D tractor-driving simulator for slip estimation-based path-tracking control of auto-guided tillage operation. Biosystems Engineering, 2019; 178: 70-85.

[18] Mizushima A, Noguchi N, Ishii K, Terao H. Position correction of vehicle inclination for agricultural vehicle guidance system. Journal of the Japanese Society of Agricultural Machinery, 1999; 61(Supp): 559-560.

[19] Inoue K, Ootsuka K, Sugimoto M, Murakami N, Li W. Sensor fusion techniques for automatic guidance by the method of Kalman filter using DGPS and gyrocompass (Part 1). Journal of the Japanese Society of
Agricultural Machinery, 1999; 61(4): 103-113. (In Japanese)

[20] Noguchi N. Agricultural vehicle robot. Journal of Robotics and Mechatronics, 2018; 30(2): 165-172.

[21] Terao H, Noguchi N, Ishii K. Development of agricultural field robot in AVSE, Hokkaido University, Japan. Proc. IV International Conference "Microprocessor Systems in Agriculture", PW Plock, Poland, 2001; pp.211-219.

[22] Mizushima A, Ishii K, Noguchi N, Matsuo Y, Lu R. Development of a low-cost attitude sensor for agricultural vehicles. Computers and Electronics in Agriculture, 2011; 76(2): 198-204.

[23] CAD Library of free DWG Blocks and premium AutoCAD drawings. 2019. Available: https://cad-block.com/. Accessed on [2020-06-27]

[24] Tofael A. Navigation of an autonomous tractor using multiple sensors. PhD dissertation. Tsukuba: University of Tsukuba, 2006; 183p.

[25] Larson D L, Smith D W, Liljedahl J B. The dynamics of three-dimensional tractor motion. Trans. of ASABE1976; 19: 195-200.

[26] Deere \& Company. John Deere Factory shipping weights and dimensions for 30 series tractors. Deere \& Company. 2013 http://www.deere.com/en US/ag/servicesupport/tips/tractors/common stor ies/pf_weights_dimensions.html. Accessed on [2020-06-29].

[27] MATLAB. Version 8.1.0.604 (R2013a). Natick, Massachusetts: The MathWorks Inc., 2013.

[28] Murphy D J. Tractor Stability and Instability. PennState Extension, 2016. https://extension.psu.edu/tractor-stability-and-instability. Accessed on [2020-06-30].

[29] Yang L, Noguchi N. Human detection for a robot tractor using omni-directional stereo vision. Computers and Electronics in Agriculture, 2012; 89: 116-125. 